\title{
Germline RBBP8 variants associated with early-onset breast cancer compromise replication fork stability
}

\author{
Reihaneh Zarrizi,, Martin R. Higgs, ${ }^{2}$ Karolin Voßgröne, ${ }^{1}$ Maria Rossing, ${ }^{3}$ Birgitte Bertelsen, ${ }^{3}$ Muthiah Bose, ${ }^{1}$ Arne Nedergaard Kousholt, \\ Heike Rösner, ${ }^{1}$ the COMPLEXO Network, ${ }^{4}$ Bent Ejlertsen, ${ }^{5}$ Grant S. Stewart, ${ }^{2}$ Finn Cilius Nielsen, ${ }^{3}$ and Claus S. Sørensen ${ }^{1}$ \\ 'Biotech Research and Innovation Centre, University of Copenhagen, Copenhagen, Denmark. ${ }^{2}$ Institute of Cancer and Genomic Sciences, College of Medical and Dental Sciences, University of \\ Birmingham, Birmingham, United Kingdom. ${ }^{3}$ Centre for Cenomic Medicine, Rigshospitalet, Copenhagen University Hospital, Copenhagen, Denmark. ${ }^{4}$ The COMPLEXO Network is detailed in Supplemental \\ Acknowledgments. ${ }^{5}$ Department of Oncology, Rigshospitalet, Copenhagen University Hospital, Copenhagen, Denmark.
}

\begin{abstract}
Haploinsufficiency of factors governing genome stability underlies hereditary breast and ovarian cancer. One significant pathway that is disabled as a result is homologous recombination repair (HRR). With the aim of identifying new candidate genes, we examined early-onset breast cancer patients negative for BRCA1 and BRCA2 pathogenic variants. Here, we focused on CtIP (RBBP8 gene), which mediates HRR through the end resection of DNA double-strand breaks (DSBs). Notably, these patients exhibited a number of rare germline RBBP8 variants. Functional analysis revealed that these variants did not affect DNA DSB end resection efficiency. However, expression of a subset of variants led to deleterious nucleolytic degradation of stalled DNA replication forks in a manner similar to that of cells lacking BRCA1 or BRCA2. In contrast to BRCA1 and BRCA2, CtIP deficiency promoted the helicase-driven destabilization of RAD51 nucleofilaments at damaged DNA replication forks. Taken together, our work identifies CtIP as a critical regulator of DNA replication fork integrity, which, when compromised, may predispose to the development of early-onset breast cancer.
\end{abstract}

\section{Introduction}

Hereditary breast and ovarian cancer (HBOC) is causally linked with germline pathogenic variants in proteins implicated in homologous recombination repair (HRR), the protection of stalled DNA replication forks, and cell cycle checkpoint control (1-6). $B R C A 1$ and BRCA2 are the most commonly mutated genes in HBOC, accounting for approximately $15 \%$ of cases (7). However, a number of less frequent genetic alterations that predispose to breast cancer have been uncovered in other genes, e.g., RECQL1, $P A L B 2$, and BRIP1 $(3,8-10)$. For the majority of emerging HBOC genes, it is currently not possible to provide accurate risk estimates, because of their rarity. This poses challenges to cancer risk management and counseling as well as burdens the families of women who carry variants in these genes. Consequently, it has been proposed that functional analyses should be employed in the classification of novel genetic variants (1).

Notably, genetic and functional analysis of breast cancerassociated variants has uncovered substantial locus heterogeneity. Loss of function of several HRR factors other than BRCA1 and BRCA2, including PALB2 and RAD51C, increases the risk of breast cancer $(1,8,11)$. CtIP, encoded by the $R B B P 8$ gene, is a major HRR factor that has thus far not been functionally linked with HBOC. CtIP is a key regulator of double-strand break (DSB) resection

Authorship note: RZ, MRH, and KV are co-first authors.

Conflict of interest: The authors have declared that no conflict of interest exists. Copyright: (5) 2020, American Society for Clinical Investigation.

Submitted: January 17, 2019; Accepted: April 22, 2020; Published: June 22, 2020.

Reference information: J Clin Invest. 2020;130(8):4069-4080.

https://doi.org/10.1172/JCl127521. operating within the BRCA1/BRCA2 pathway, and generates the single-stranded DNA needed for RAD51-mediated recombination. Here, we examined a high-risk population of early-onset $B R C A 1$ and BRCA2 mutation-negative breast cancer patients for germline variants in $R B B P 8$. Compared with a Danish control cohort, these patients were enriched for a subset of rare $R B B P 8$ variants. Functional analysis revealed that while these CtIP variants did not affect DSB resection efficiency, their expression led to deleterious nucleolytic degradation of stalled replication forks in a manner similar to that of cells lacking BRCA1/BRCA2. Notably, CtIP deficiency promoted the helicase-driven destabilization of RAD51 nucleofilaments at damaged replication forks. Taken together, our work identifies CtIP as a critical regulator of replication fork integrity that, when mutated, may predispose to the development of early-onset breast cancer.

\section{Results}

Identification of RBBP8 germline variants. We first screened a group of 129 Danish high-risk $B R C A 1$ and $B R C A 2$ pathogenic variantnegative breast cancer patients for germline variants in $R B B P 8$ (patient group I, outlined in Supplemental Figure 1; supplemental material available online with this article; https://doi.org/10.1172/ JCI127521DS1). Fifty percent of women had a first- or seconddegree relative with breast or ovarian cancer, including women below 35 years of age at the time of diagnosis, male breast cancer patients, and 6 women with early-onset ovarian cancer (Supplemental Table 1). This initial screening identified 5 different non-synonymous, heterozygous RBBP 8 variants (Table 1 and Figure $1, \mathrm{~A}$ and B). Three patients were carriers of an in-frame 3-bp deletion in exon 18 (c.2410_2412del; p.E804del), which was detected 
A

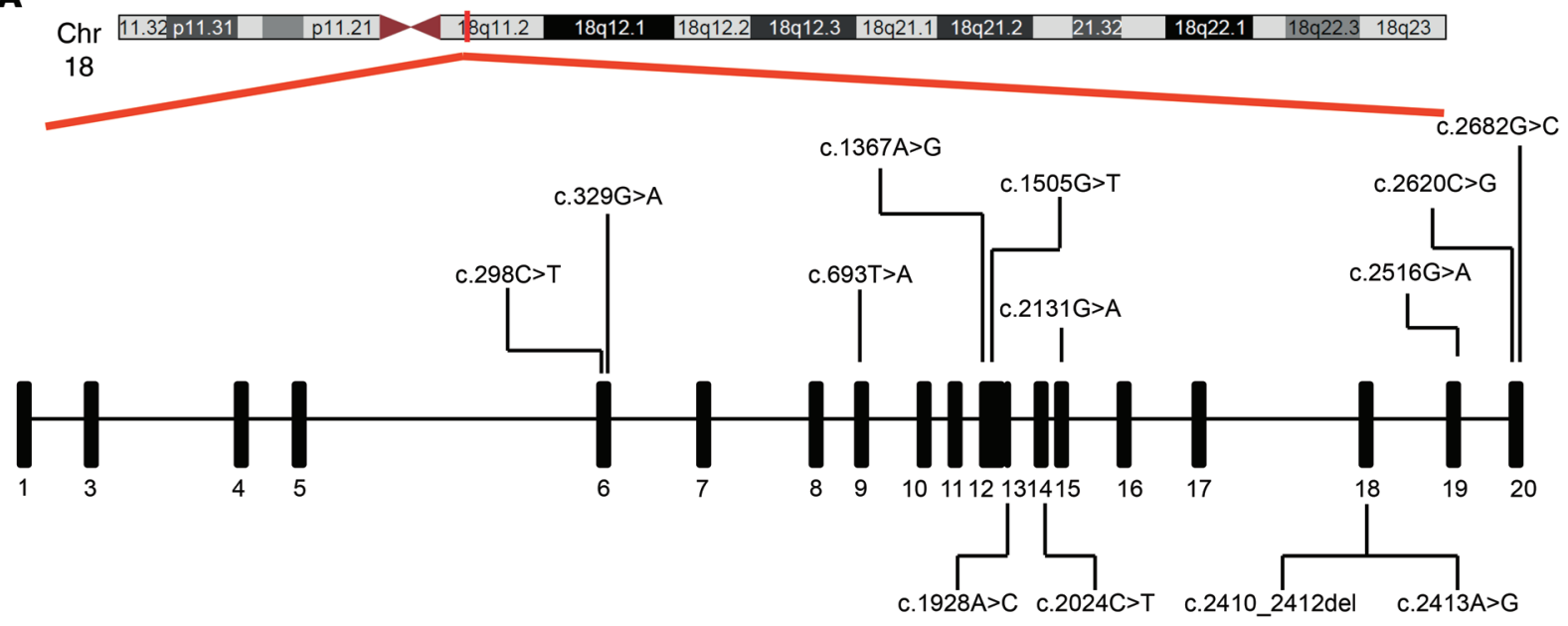

B

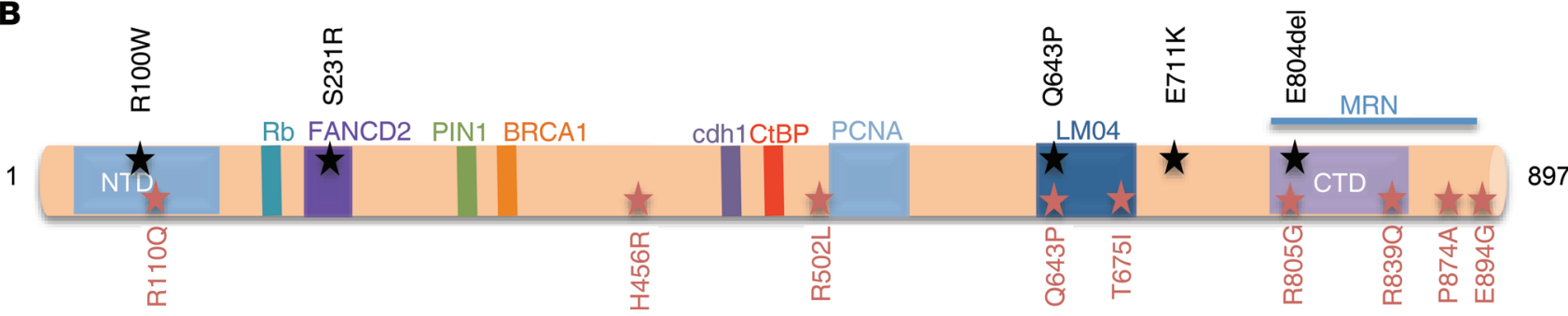

Figure 1. Identification of RBBP8 germline variants. (A) Schematic representation of the identified variants at the gene level indicated according to exon location. (B) Schematic representation of the identified variants at the protein level positioned according to known functional domains in CtIP. The multimerization domain (aa 45-165, NTD), the Sae2-like domain (aa 790-897, CTD), and identified CtIP interaction sites with other proteins are indicated. Identified variants are indicated with stars: black stars denote patient group I and red stars denote patient group II variants.

at an allele frequency of $1.16 \%$. The p.E804del variant is significantly overrepresented in our cohort with respect to 2000 Danes (12). In addition, 2 patients were carriers of different missense $R B B P 8$ variants (c.693T $>$ A, p.S231R in exon 9, and c.1928A $>$ C, p.Q643P in exon 13, respectively), and 1 patient carried 2 different missense variants (c.298C > T, p.R100W in exon 6, and c.2131G>A, p.E711K in exon 15). Only the p.R100W variant was detected in 2000 Danes, while neither the p.Q643P nor the p.E711K variant had, to our knowledge, been reported previously (12).

We subsequently sequenced $R B B P 8$ in a larger series of 1092 patients negative for $B R C A 1$ and $B R C A 2$ pathogenic variants with breast cancer and/or ovarian cancer or other related cancer types, as well as unaffected individuals of families with HBOC (patient group II, outlined in Supplemental Figure 1). Nine different heterozygous missense variants in RBBP\& (Table 1 and Figure 1, A and B) were identified in 14 females from this cohort. Three patients carried a p.R110Q variant, 2 carried a p.H456R variant, and 3 carried the p.Q643P variant previously identified among patient group I. A further 6 variants - p.R502L, p.T675I, p.R805G, p.R839Q, p.P874A, and p.E894D - were identified in individual patients. In total, we identified $13 \mathrm{RBBP} 8$ variants in 21 patients (Table 1), 9 of which were observed once. Finally, we explored an international cohort of 1054 breast cancer patients without pathogenic variants in $B R C A 1$ or $B R C A 2$ for rare variants in $R B B P 8$. Here, we identified 17 different rare variants in 22 patients, of whom the clinically annotated $(n=7)$ had a median age of 38 at the time of diagnosis (Supplemental Table 2). These $R B B P 8$ variants also included the p.Q643P variant and 2 loss-offunction variants (Supplemental Table 2).

RBBP8/CtIP variants display a genome maintenance defect. Since our genetic screening indicated that $R B B P 8$ variants could be associated with early-onset breast cancer, we investigated whether they affect known CtIP function(s). Hence, we examined DNA DSB end resection as well as genome stability after exposure to irradiation (IR) or replication stress induced with aphidicolin (APH) or hydroxyurea (HU). To create an isogenic system for our assays, we first depleted endogenous RBBP8/ CtIP from breast cancer MCF7 cells with siRNA. We then complemented cells with re-expression of siRNA-resistant CtIP variants (Figure 2, A and B). All variants were well expressed; surprisingly, however, none of these variants affected the ability of cells to perform DNA DSB end resection after IR (Figure 2C and Supplemental Figure 2A). The phosphorylation of RPA32 on the S4/S8 residues was used as a readout for the proficiency of DNA DSB end resection after IR in these assays (13). Next, we monitored genome stability after exposure to IR, APH, or $\mathrm{HU}$, using the accumulation of extranuclear micronuclei as a readout (Supplemental Figure 2B). Similarly to WT-CtIP, and in keeping with our findings above, all the tested variants were able to complement the IR-induced genome instability caused 
Table 1. Identified RBBP8 variants and allele frequencies

\begin{tabular}{|c|c|c|c|c|c|c|c|c|c|c|}
\hline $\begin{array}{l}\text { Nucleotide } \\
\text { (HCVS) }\end{array}$ & $\begin{array}{l}\text { Protein } \\
\text { (HGVS) }\end{array}$ & Exon & Group I & Group II & $\begin{array}{c}\text { AF group I } \\
(\%)\end{array}$ & $\begin{array}{c}\text { AF group I } \\
\text { II (\%) }\end{array}$ & $\begin{array}{c}\text { + AF controls } \\
(\%)\end{array}$ & AF NFE (\%) & $\begin{array}{c}P \text { value AF } \\
\text { (group I vs. } \\
\text { controls) }\end{array}$ & $\begin{array}{l}\text { P value AF } \\
\text { (group I + II } \\
\text { vs. controls) }\end{array}$ \\
\hline c. $298 \mathrm{C}>\mathrm{T}$ & p.R100W & 6 & 1 & & 0.388 & 0.041 & 0.025 & 0.008 & NS & NS \\
\hline c.693Т >A & p.S231R & 9 & 1 & & 0.388 & 0.041 & - & - & NS & NS \\
\hline c. $1367 A>C$ & p.H456R & 12 & & 2 & - & 0.082 & 0.127 & 0.220 & - & NS \\
\hline c. $1505 G>T$ & p.R502L & 12 & & 1 & - & 0.041 & 0.025 & 0.003 & - & NS \\
\hline c. $2024[>T$ & р.T675I & 14 & & 1 & - & 0.041 & - & 0.011 & - & NS \\
\hline c. $2131 \mathrm{G}>\mathrm{A}$ & p.E711K & 15 & 1 & & 0.388 & 0.041 & - & - & NS & NS \\
\hline c.2410_2412del & p.E804del & 18 & 3 & & 1.163 & 0.123 & - & 0.015 & 0.0002 & NS \\
\hline c. $2413 A>G$ & p.R805G & 18 & & 1 & - & 0.041 & - & 0.003 & - & NS \\
\hline c. $2516 \mathrm{G}>\mathrm{A}$ & p.R839Q & 19 & & 1 & - & 0.041 & - & 0.088 & - & NS \\
\hline c. $2620 C>C$ & p.P874A & 20 & & 1 & - & 0.041 & - & 0.008 & - & NS \\
\hline
\end{tabular}

AF, allele frequency; HCVS, Human Genome Variation Society nomenclature; NFE, non-Finnish European in gnomAD. Fisher exact test for AF in group I and group I + II compared with AF in controls (2000 Danish individuals; ref. 12).

by the loss of CtIP (Table 2). Together, these data indicate that the identified germline $R B B P 8$ variants do not give rise to a detectable impairment of DNA DSB repair. However, expression of several variants (Q643P, E804del, and R805G) as well as a C-terminal-truncated CtIP $(\Delta \mathrm{C})$ mutant failed to complement the genome instability induced by APH and HU following depletion of endogenous CtIP (Table 2). This suggests that these variants perturb a function of CtIP specifically associated with the replication stress response. In addition to the Danish breast cancer cohort, we also investigated the $R B B P 8$ variants present in the international COMPLEXO cohort using similar methods. The CtIP-Q643P variant, as well as the truncating variants CtIP-R185* and CtIP-L372*, displayed increased genome instability after replication stress (Supplemental Table 3).

CtIP-E804del is proficient in HRR. To further examine the potential HRR status of CtIP variants in the Danish breast cancer cohort, we focused on the CtIP-E804del variant, as it was significantly enriched in this cohort. We used the tractable U-2OS cell line, which is commonly used to evaluate CtIP function $(14,15)$, and generated an inducible complementation system expressing siRNA-resistant GFP-tagged full-length CtIP or the CtIP-E804del variant (Supplemental Figure 2, C-H). Consistent with our previous results in MCF7 cells (Figure 2C), expression of the CtIP-E804del variant in U-2-OS cells could rescue the DSB resection deficiency resulting from CtIP depletion (Supplemental Figure 2, C and D). We then set out to assess HRR efficiency in CtIP-E804del U-2-OS cells, since CtIP-dependent DSB end resection is crucial for efficient HRR. As expected, expression of CtIP-E804del variant could rescue the HRR deficiency caused by CtIP depletion (Supplemental Figure 2, E-G). Since HRR deficiency can be therapeutically exploited through the use of PARP inhibitors (PARPi), we also investigated whether CtIP-E804del variant expression promotes PARPi sensitivity. As shown in Supplemental Figure 2H using variant complementation of siRNA-depleted cells, the CtIP-E804del variant did not display any increase in PARPi sensitivity over and above WT-CtIP-complemented cells. Taken together, these results indicate that the CtIP-E804del variant displayed proficient DSB end resection and HRR. Furthermore, this suggests that CtIP variants deficient in responding to replication stress may promote tumorigenesis independently of HRR.

CtIP promotes RAD51 function during replication stress. In order to functionally characterize a subset of variants in greater detail, the CtIP-Q643P and CtIP-E804del variants were chosen because they were significantly enriched in our Danish breast cancer cohort and were associated with increased genome instability upon HU and APH treatment (Table 2). Additionally, the CtIPR805G variant was also chosen because of its close amino acid sequence proximity to the CtIP-E804del variant and its defective response to replication stress (Table 2).

Notably, Q643P, E804del, and R805G CtIP variants could not be linked with deficiency in DNA end resection. As an alternative explanation underlying their functional contribution, we hypothesized that these variants may instead be deficient in replication fork stabilization, which is a recently emerging function of CtIP (16). To test this hypothesis, we first analyzed the prevalence of RPA foci. This is a robust marker of ssDNA accumulating at replication forks after $\mathrm{HU}$ treatment, with both increases and decreases in the number of RPA foci per cell being indicative of a perturbed replication stress response. Consistent with previous reports (17), CtIP depletion led to an increase in HU-induced RPA foci formation, which could be rescued by expression of exogenous WT-CtIP-GFP (Figure 3, A and B). Interestingly, this was not the case for the CtIP-E804del or CtIP-R805G variants (Figure 3, A and B). Serving as negative control, variants that did not display genomic instability after replication stress could also rescue the elevated level of RPA foci formation resulting from CtIP knockdown (Supplemental Figure 3A). Intriguingly, the CtIP-Q643P variant suppressed RPA in a manner comparable to that of WT-CtIP (Figure 3, A and B). 
A

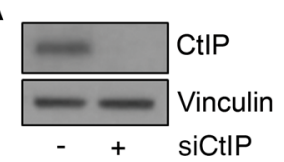

B

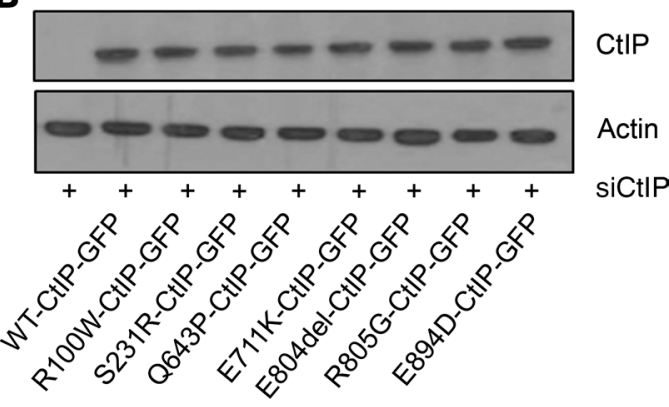

In order to directly visualize RAD51 recruitment to the stalled forks after HU treatment, we turned to isolation of proteins on nascent DNA (iPOND), using click chemistry to conjugate biotin to a nucleoside analog (EdU) incorporated into newly synthesized DNA (19). Our analyses primarily focused on comparing WT-CtIP with the CtIP-E804del variant, since this variant exhibited a defective response to replication stress. Using iPOND, the recruitment of RAD51 to nascent DNA damaged with $\mathrm{HU}$ was reduced in the absence of CtIP, in agreement with our previous data (Figure 3, C and D). Moreover, this deficiency was restored by the complementation with WT-CtIP. Importantly, however, this was not the case after complementation with the CtIPE804del mutant (Supplemental Figure 3C). To understand whether CtIP is recruited directly to stalled forks after HU treatment, we used a proximity ligation assaybased (PLA-based) approach that measures the association of proteins on nascent DNA $(20,21)$. Following the depletion of CtIP from U-2-OS cells, the expression of WT-CtIP or CtIP-E804del was induced in cells with doxycycline. Cells were then labeled with EdU for 10 minutes before treatment with $4 \mathrm{mM}$ HU for 5 hours. Click chemistry was then used to conjugate biotin to EdU, and PLA was conducted to detect protein binding to biotinlabeled nascent DNA. Using this approach, our data revealed that WT-CtIP was present at nascent DNA after replication stress, while CtIP-E804del was absent under the same conditions (Supplemental Figure 3, D and E).

Together, these data suggest that CtIP prevents the proficiency. (A and B) Western blot analysis of extracts from MCF7 cells transfected with the indicated siRNA (A), and coexpression of siRNA-resistant GFP-tagged CtIP variants (B). Actin and vinculin were used as loading controls. (C) The relative intensity of phosphorylated RPA32 (S4/8) was examined 3 hours after exposure of MCF7 cells to IR. Cells were fixed and stained for p-RPA (S4/8). Each of the variants was compared with WT-CtIP-GFP, but no significant changes were observed. The displayed data represent 3 independent biological replicates, and $n \geq 280$ nuclei were analyzed per sample. Holm-corrected multiple testing was performed of ranked data fitted by a linear mixed model, comparing all CtIP variants with WT-CtIP-GFP. ${ }^{* *} P<0.0001$.

To obtain insight into the underlying mechanisms, we further examined how CtIP-depleted cells responded to replication stress. Since RAD51 nucleofilaments protect stalled replication forks from uncontrolled nucleolytic degradation $(5,18)$, we addressed whether CtIP affects RAD51 localization at damaged forks. As shown in Figure 3, C and D, HU-induced RAD51 foci formation was reduced in MCF7 cells depleted of CtIP. Notably, expression of neither the E804del nor the R805G CtIP variants could complement the loss of HU-induced RAD51 foci formation caused by CtIP depletion (Figure 3, C and D, and Supplemental Figure 3B), while this could be restored by transient expression of WT-CtIP and several other potentially nonpathogenic CtIP variants. These data therefore suggest that the Sae2-like domain of CtIP might play a role in recruiting/stabilizing RAD51 after replication stress. Intriguingly, the HU-induced RAD51 response was comparable in cells expressing the CtIP-Q643P variant and cells expressing WT-CtIP (Figure 3, C and D), which suggests that this variant promotes replication stressinduced genome instability via another mode of action. accumulation of ssDNA at damaged replication forks by recruiting/stabilizing RAD51 and that the cancer-associated CtIP variant E804del compromises this function.

CtIP antagonizes the excessive degradation of stalled replication forks through FBH1. Since RAD51 is known to protect stalled replication forks from degradation and loss of CtIP causes a decrease in RAD51 foci formation, we sought to measure replication fork degradation directly, using the single-molecule DNA fiber-based assay $(18,22)$. CtIP was depleted from U-2-OS cells, and expression of WT-CtIP and the E804del or $\Delta \mathrm{C}$ variants was induced. These cells were then sequentially pulse-labeled with CldU and IdU to label nascent DNA before prolonged fork stalling with $\mathrm{HU}$ (Figure 4A). In keeping with previous reports (23), these analyses showed that loss of CtIP results in increased degradation of nascent DNA at stalled replication forks (Figure 4B). Moreover, this was abolished upon the expression of WT-CtIP, but not by expression of the $\Delta \mathrm{C}$ mutant (Figure $4 \mathrm{C}$ ). Importantly, the E804del variant was partially deficient in replication fork protection after $\mathrm{HU}$ (Figure 4C). Thus, we surmise that the role of CtIP in preventing nascent DNA degradation at stalled forks involves its C-terminal Sae2-like domain.

Finally, we asked whether CtIP plays a role in recruiting RAD51 to stalled forks in a manner similar to that of BRCA1/2, or stabilizing RAD51 at these structures like BOD1L and WRNIP1. Unlike BRCA1/2, BOD1L and WRNIP1 protect damaged forks by suppressing the anti-recombinase activity of proteins such as FBH1 and BLM $(15,20)$. Moreover, it has been shown that loss 
Table 2. CtIP suppresses genomic instability at perturbed replication forks

\begin{tabular}{|c|c|c|c|c|c|c|c|c|}
\hline \multirow[b]{2}{*}{ CtIP variants } & \multicolumn{2}{|c|}{ Cytochalasin B } & \multicolumn{2}{|c|}{ IR } & \multicolumn{2}{|c|}{ APH } & \multicolumn{2}{|c|}{$\mathrm{HU}$} \\
\hline & $\begin{array}{l}\% \text { of binuclei with } \\
\text { micronuclei }\end{array}$ & $P$ value & $\begin{array}{l}\% \text { of binuclei with } \\
\text { micronuclei }\end{array}$ & $P$ value & $\begin{array}{l}\% \text { of binuclei with } \\
\text { micronuclei }\end{array}$ & $P$ value & $\begin{array}{l}\% \text { of binuclei with } \\
\text { micronuclei }\end{array}$ & $P$ value \\
\hline Vector (GFP) & 49.95 & & 67.30 & & 66.67 & & 67.14 & \\
\hline WT & 47.37 & - & 46.46 & - & 47.96 & - & 48.36 & - \\
\hline R1100W & 45.13 & NS & 48.38 & NS & 47.53 & NS & 47.39 & NS \\
\hline R110Q & 46.46 & NS & 47.99 & NS & 49.31 & NS & 50.18 & NS \\
\hline S231R & 47.09 & NS & 48.29 & NS & 49.29 & NS & 47.36 & NS \\
\hline H456R & 47.76 & NS & 49.76 & NS & 47.99 & NS & 45.34 & NS \\
\hline R502L & 48.77 & NS & 49.43 & NS & 50.12 & NS & 50.14 & NS \\
\hline $\mathrm{R} 589 \mathrm{H}$ & 48.99 & NS & 48.04 & NS & 48.77 & NS & 47.70 & NS \\
\hline Q643P & 49.33 & NS & 48.45 & NS & 60.74 & 0.0001 & 57.83 & 0.0030 \\
\hline E711K & 46.35 & NS & 48.03 & NS & 45.47 & NS & 48.20 & NS \\
\hline E804del & 49.34 & NS & 47.99 & NS & 65.32 & 0.0001 & 69.32 & 0.0001 \\
\hline R805G & 51.62 & NS & 48.94 & NS & 66.67 & 0.0001 & 68.15 & 0.0001 \\
\hline R839Q & 49.79 & NS & 47.60 & NS & 49.12 & NS & 48.28 & NS \\
\hline P874A & 48.72 & NS & 48.89 & NS & 50.20 & NS & 48.53 & NS \\
\hline E894D & 47.46 & NS & 47.39 & NS & 49.09 & NS & 49.17 & NS \\
\hline$\Delta C$ & 50.37 & NS & 61.26 & 0.0001 & 59.38 & 0.0002 & 58.78 & 0.0010 \\
\hline
\end{tabular}

MCF7 cells were transfected with the indicated siRNA followed by transfection of WT or its mutated CtIP variants. Further, cells were treated with IR or the indicated dose of APH for 16 hours or $4 \mathrm{mM} \mathrm{HU}$ for 5 hours and cytochalasin $B$ for 36 hours. DAPI stain was used to visualize nuclei. Cells were imaged with a $\times 20$ objective on a scanR workstation (Olympus). At least 100 green cells were counted for each genotype per experiment. One-way ANOVA with Dunnett's multiple-comparisons test was performed on 3 independent replicates. All variants were compared with WT-CtIP-GFP.

of the anti-recombinase FBH1 increases RAD51 foci formation at stalled replication forks (24). Therefore, we hypothesized that FBH1 might be involved in evicting RAD51 from stalled forks in the absence of CtIP. In keeping with this prediction, concomitant depletion of FBH1 and CtIP rescued RAD51 accumulation in HU-treated conditions to control levels (Figure 4, D and E). To further explore the link between CtIP and FBH1, we performed fork degradation assays in HU-treated cells depleted of CtIP, FBH1, or CtIP and FBH1 together (Figure 4F). These experiments revealed that loss of FBH1 restored nascent DNA stability in the absence of CtIP (Figure 4F), suggesting that CtIP stabilizes RAD51 nucleofilaments to suppress fork degradation. Depletion of FBH1 in cells expressing the CtIP-E804del variant also restored nascent strand stability, and restabilized RAD51 at stalled replication forks (Figure 4, G and H, and Supplemental Figure 4, A and B). These data therefore suggest that CtIP regulates replication fork stability by suppressing FBH1-mediated eviction of RAD51 from stalled forks, and that cancer-associated mutations in the C-terminus of CtIP perturb this vital function (Figure $4 \mathrm{H}$ ).

\section{Discussion}

Our study demonstrates a role for rare $R B B P 8$ variants in the control of DNA replication fork integrity. Altogether we identified 13 $R B B P 8$ germline variants in 21 patients, of whom 3 patients had the C-terminal E804del variant. Importantly, we identified 3 RBBP8 variants that were associated with increased genome instability. These variants were located in the C-terminus (E804del and R805G) and LMO4-interacting (Q643P) regions of CtIP. The $\mathrm{C}$-terminus region is crucial for CtIP functions in genome maintenance, and consistent with this, localization of RAD51 and RPA to sites of damage was impaired by expression of the E804del and R805G variants. Regarding the variant in the LMO4-interacting region (Q643P), although the functional role of this domain is unclear, it is conceivable that the breast cancer risk associated with this variant may relate to the dysregulation of LMO4. However, we were unable to detect a variant-dependent interaction between CtIP and LMO4 (Supplemental Figure 4C). Further studies of this variant may identify additional roles for CtIP in maintaining genome stability and suppressing cancer susceptibility.

Surprisingly, those CtIP variants that promoted genome instability were functionally WT for DNA DSB end resection and HRR. Instead, we demonstrate that CtIP protects stalled replication forks against enhanced fork degradation by promoting RAD51 nucleofilament stability, and it is this function that is perturbed by variants associated with early-onset breast cancer. Thus, these results suggest that CtIP insufficiency may predispose to breast cancer by allowing deleterious replication fork degradation (Figure 4I). Interestingly, loss of fork protection is a potential target for cancer therapy, since the ability of BRCA1/2-deficient cells to acquire drug resistance is intimately linked to fork protection (6).

A pathway protecting stalled DNA forks from degradation was first uncovered in cells with BRCA2 insufficiency, and more recently has been reported in cells lacking critical tumor suppressors known to be involved in regulating HRR, including BRCA1, PALB2, and FANCD2 $(6,18,21,25)$. Our research now links CtIP with these factors that facilitate stable accumulation of RAD51 when forks are challenged. However, we suggest that CtIP, unlike BRCA1, BRCA2, and PALB2, belongs to a family of replication fork protection factors, including BOD1L and WRNIP1, which regulate the FBH1 helicase, a RAD51-evicting 
A

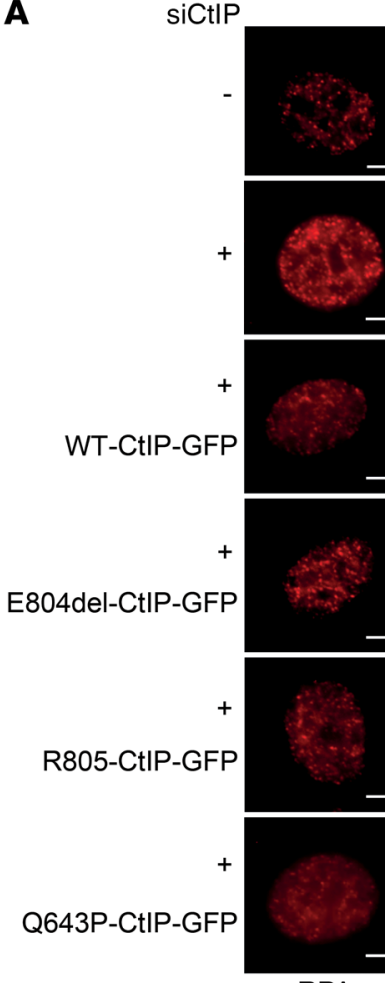

RPA
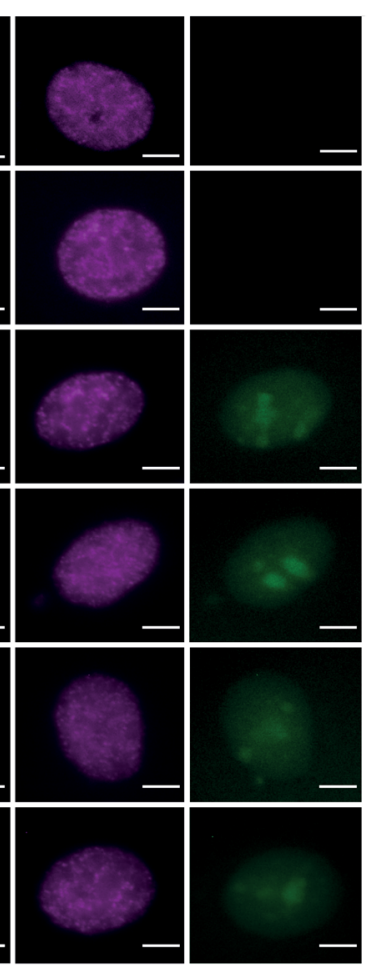

EdU

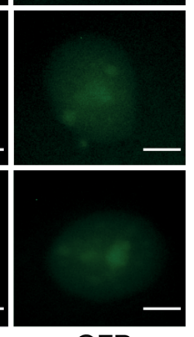

GFP

D

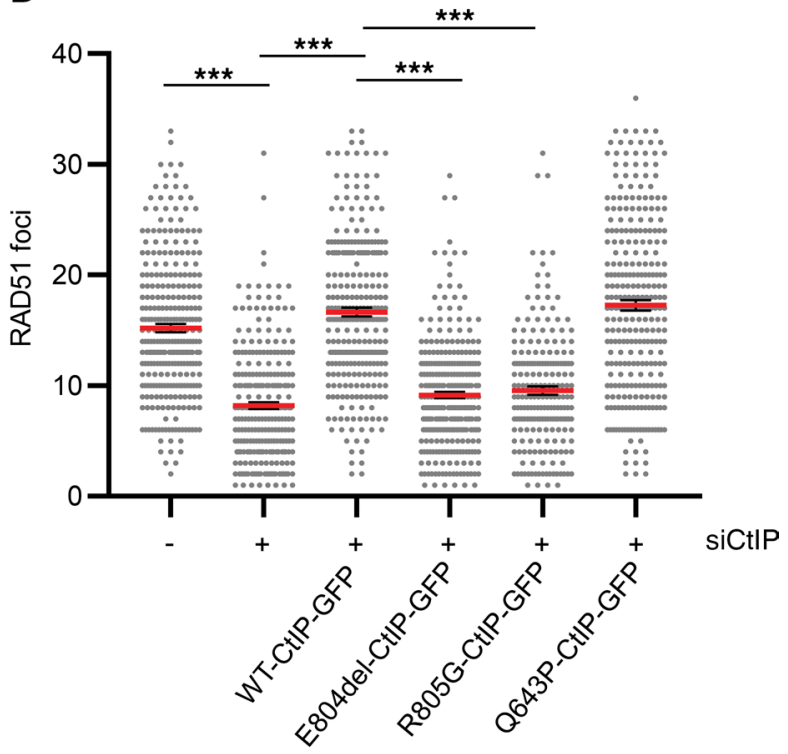

C

E804del-CtIP-GFP
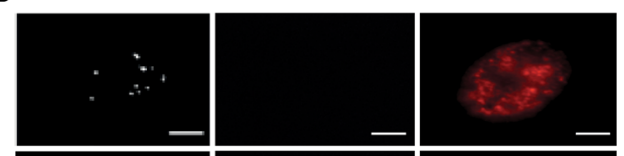

WT-CtIP-GFP
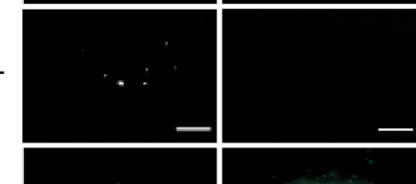

$+$

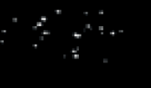

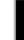
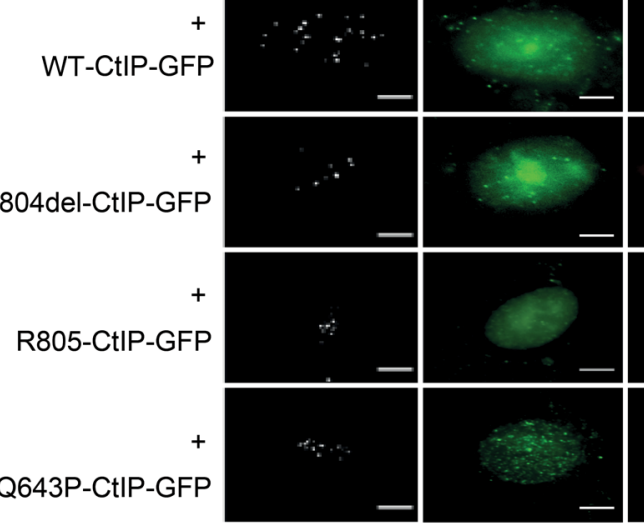

RAD51

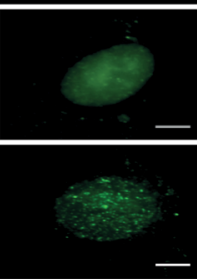

GFP
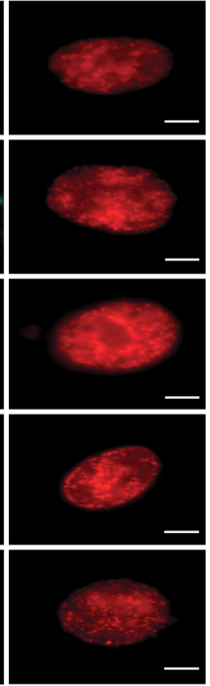

EdU
B
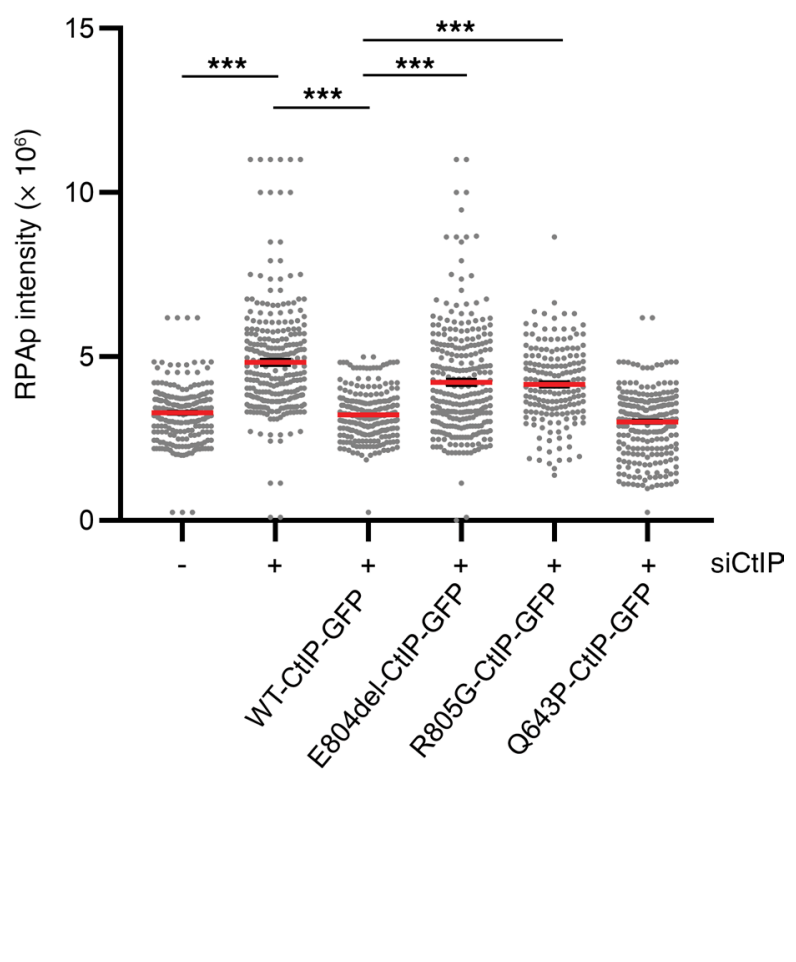

Figure 3. CtIP prevents ssDNA accumulation after replication stress. (A) Representative images displaying RPA in HU-treated EdU+ cells. Scale bars: 20 $\mu \mathrm{m}$. (B) MCF7 cells were transfected with the indicated siRNA, and 24 hours later, cells were transfected with WT or its mutated CtIP variants. Afterward, cells were pulsed with $10 \mu \mathrm{M}$ EdU for 20 minutes before addition of $4 \mathrm{mM} \mathrm{HU}$. Cells in S phase (EdU+) at the time of HU treatment were Click-iT-labeled (Thermo Fisher Scientific) with an Alexa Fluor 594 azide, and RPA intensity in EdU+ cells was enumerated using Imagej/Fiji. The displayed data represent 3 independent biological replicates, and $n \geq 174$ nuclei were analyzed per sample. Holm-corrected multiple testing was performed of ranked data fitted by a linear mixed model, comparing all CtIP variants with WT-CtIP-GFP. ${ }^{* * *} P<0.0001$. (C) Representative images displaying RAD51 in HU-treated EdU+ cells. Scale bars: $20 \mu \mathrm{m}$. (D) MCF7 cells were transfected with the indicated siRNA, and 24 hours later, cells were transfected with WT or mutated CtIP variants. Afterward, cells were pulsed with $10 \mu \mathrm{M}$ EdU for 20 minutes before addition of $4 \mathrm{mM} \mathrm{HU}$. Cells in S phase (EdU') at the time of HU treatment were ClickIT-labeled with an Alexa Fluor 594 azide, and RAD51 foci in EdU+ cells were enumerated using Image//Fiji. The displayed data represent 3 independent biological replicates, and $n \geq 207$ nuclei were analyzed per sample. Holm-corrected multiple testing was performed of ranked data fitted by a linear mixed model, comparing all CtIP variants with WT-CtIP-GFP. 
factor. Thus, in the absence of these RAD51-stabilizing factors, FBH1 reduces the presence of RAD51 at stalled forks, allowing uncontrolled fork degradation that can trigger genome instability. This is an emerging biological response to fork stalling, and the links with tumorigenesis are only now starting to be dissected. Notably, our functional findings on CtIP are in agreement with recently published data that also identify a role for CtIP in suppressing degradation of stalled replication forks (16). The authors of this study focused on the role of the N-terminal region of CtIP that helps to minimize degradation of forks by the DNA2 nuclease. Thus far, we have not identified cancer-associated disabling variants in this CtIP region.

The roles of CtIP in breast cancer predisposition and progression are not well understood, though studies have indicated that a lack or low levels of CtIP expression in tumor cells is associated with a reduced survival rate $(23,26)$. Furthermore, tumors lacking CtIP display an impaired ability to repair DSBs, which leads to increased sensitivity to PARP inhibitors $(26,27)$. Thus, determining the impact of identified variants in CtIP on its function should be considered in trying to personalize a therapeutic approach for treating a specific patient. Intriguingly, analysis of a cohort of $129 B R C A 1$ and $B R C A 2$ mutation-negative Australian breast cancer patients failed to demonstrate an enrichment of coding variants in $R B B P 8$ (28). In fact, no coding $R B B P 8$ variants were identified except for a polymorphism in intron 6. In contrast, a recent Spanish study identified 2 truncating $R B B P 8$ variants in 2 early-onset $B R C A 1 / 2$ mutation-negative breast cancer patients (29). Furthermore, we identified 2 functionally damaging truncating variants in the COMPLEXO cohort in addition to the Q643P variant also described here. The differences between studies may reflect population differences, cohort sizes, and age of breast cancer onset in the cohorts.

Murine studies have indicated that CtIP haploinsufficiency is tumor-promoting, whereas a complete loss of CtIP is detrimental, leading to inviability of mice (30). In contrast, murine tissuespecific conditional CtIP ablation systems indicated that a complete loss of CtIP suppresses tumorigenesis (31). However, it is likely that these observations are due to a deleterious decrease in cellular fitness linked to a complete loss of HRR. Importantly, we have shown that a subset of $R B B P 8$ variants identified in this study are hypomorphic in a manner by which they impair some functions of CtIP, but not all. Based on this, we propose that hypomorphic but not lossof-function mutations in RBBP8 predispose to early-onset breast cancer. We cannot exclude the possibility that these variants may represent rare variants with little association with cancer development. However, it is noteworthy that we identified RBBP8 germline variants in early-onset breast cancer patients at a frequency similar to that previously reported for $\mathrm{HBOC}$-associated mutations in BRIP1, RECQL1, and PALB2 (3, 8-10). Thus, since our data indicate that $R B B P 8$ variants are more frequent in early-onset breast cancer cases than in unaffected population-matched controls, this frequency warrants consideration of including $R B B P 8$ in gene panels when carrying out sequencing studies on predisposition to breast cancer. Finally, our study shows the usefulness of combining genetic screening in a high-risk phenotype with comprehensive variant-centered functional analysis to identify and classify new variants implicated in hereditary cancer syndromes.

\section{Methods}

Patients. All patient samples were consecutively received for HBOC diagnostics over a period of 20 years, according to the contemporary national HBOC guidelines (Danish Breast Cancer Cooperative Group [DBCG]). Clinical and histopathological data were retrieved from the Danish Pathology Registry and the DBCG registry. Patients were diagnosed between the years 1978 and 2016.

Patient group I included 129 breast and/or ovarian cancer patients, previously identified as $B R C A 1 / 2$ pathogenic variant-negative as part of their diagnostic workup (124 females and 5 males). Female patients were diagnosed with ovarian or breast cancer at the age of 35 years or younger, while male breast cancer patients were included regardless of age at time of diagnosis. Among the female patients, 116 had breast cancer only, 6 had ovarian cancer only, 1 had breast and ovarian cancer, and 1 had breast and cervical cancer. Four of the men had breast cancer only, whereas 1 had both breast and prostate cancer. Mean age at time of diagnosis was 30 years for the female patients and 59 years for the male patients.

Patient group II included 1092 BRCA1/2-negative samples from unselected and consecutive patients undergoing genetic screening for HBOC according to clinical guidelines as described above.

Sequencing of RBBP8 in patient group I. Genomic DNA was purified from peripheral blood samples, and library preparation was performed using SeqCap EZ Human Exome Library v3 (Roche NimbleGen) or SureSelect All Exon kit v5 (Agilent Technologies) following the manufacturers' instructions. Sequencing was conducted using the HiSeq2500 or NextSeq500 platforms from Illumina. The average coverage of all exomes was $65 \times$.

Data processing. Fastq files were processed using CLC Biomedical Genomics Workbench v3 (QIAGEN). Reads were mapped to the human reference genome hg19/GRCh37, and variant calling was performed by a maximum likelihood approach on a Bayesian model. Variants were called with a minimum of 10 reads, 3 counts, and a frequency of $25 \%$. Called variants were filtered using Ingenuity Variant Analysis (http://ingenuity.com). First, variants with call quality under 20 and read depth under 10 were disregarded. Second, variants with an allele frequency of more than $1 \%$ in a public variant database, including the 1000 Genomes Project (www.1000genomes.org) or the Genome Aggregation Database (gnomAD; http:// gnomad.broadinstitute.org), were excluded unless established as a pathogenic common variant. Third, only coding non-synonymous variants and splice site variants $( \pm 2 \mathrm{bp})$ were kept. Finally, output was filtered to include the RBBP8 gene. Samples $(n=1054)$ from the COMPLEXO consortium were initially processed from raw fastq reads and aligned to the human genome reference (hg19) using the Burrows-Wheeler Aligner (v0.5.9) (32) on a per-lane basis. Alignment file preprocessing and germline variant calling were performed by the Genome Analysis Toolkit (GATK; v3.1-144). HaplotypeCaller algorithm was used to generate variant files (.vcf), which were filtered to include only rare variants in the RBBP8 gene ( $<1 \%$ in ExAC).

Sequencing of RBBP8 in patient group II. Screening of the larger group for $R B B P 8$ variants was performed using a gene panel. The library was designed to capture all exons as well as the first and last $50 \mathrm{bp}$ of the intronic regions. Samples were pooled into groups of 4 and deeply sequenced (average coverage of 5.500×). Mapping and variant calling were done as described for patient group I; however, as samples were pooled into groups of 4 , variants were called with a minimum of 100 


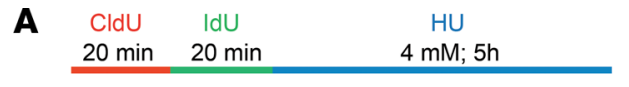

B
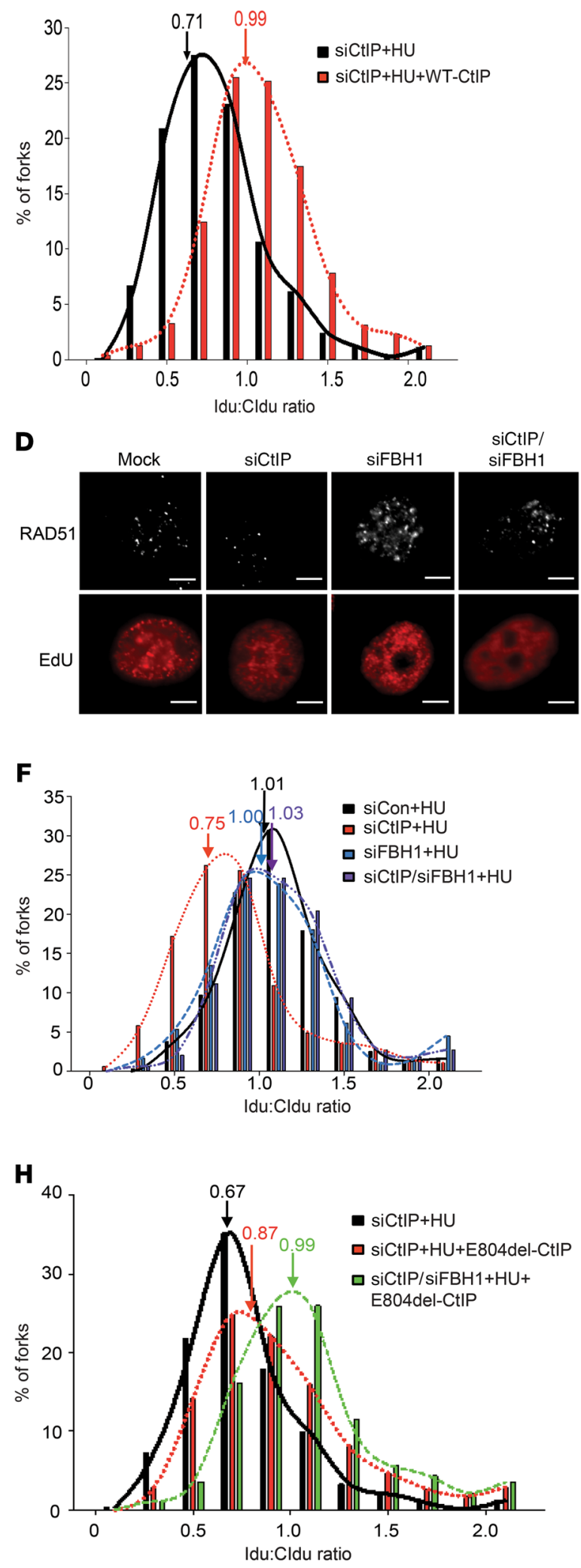
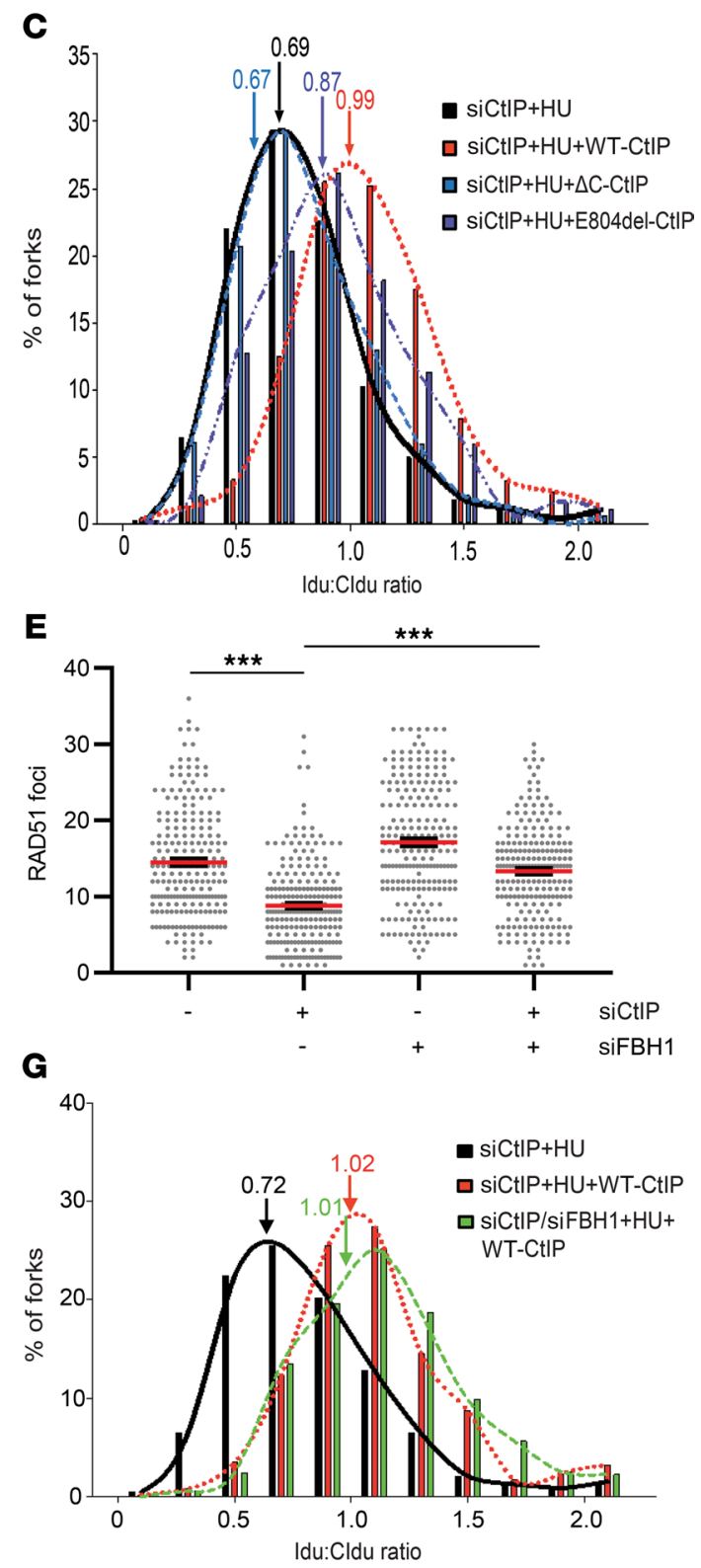

I

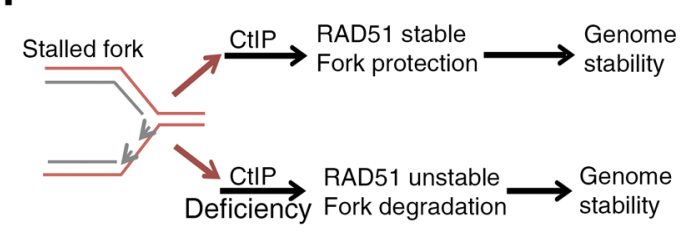


Figure 4. CtIP promotes replication fork protection through FBH1. (A) Experimental scheme of dual labeling of DNA fibers in doxycycline-inducible U-2-OS cells stably expressing the siRNA-resistant full-length WT, E804del, or $\Delta C$ CtIP. Cells were sequentially pulse-labeled with CldU and IdU, then treated with $4 \mathrm{mM} \mathrm{HU}$ for 5 hours. (B and C) Loss of CtIP results in replication fork instability in response to replication stress. Doxycycline-inducible U-2-OS cells were transfected with CtIP siRNA, and 24 hours later, induced with doxycycline for 24 hours. IdU/CldU rations are given. (D and E) MCF7 cells were transfected with the indicated siRNAs. Cells were pulsed with 10 $\mu \mathrm{M}$ EdU for 20 minutes before addition of $4 \mathrm{mM} \mathrm{HU}$. Cells in S-phase (EdU+) at the time of HU treatments were Click-IT-labeled with an Alexa Fluor 594 azide. (D) Representative images of RAD51 foci. (E) RAD51 foci in EdU ${ }^{+}$cells were enumerated using Image//Fiji. The displayed data represent 3 independent biological replicates, and $n=224$ nuclei were analyzed per sample. Holm-corrected multiple testing was performed of ranked data fitted by a linear mixed model. ${ }^{* * *} P<0.0001$. (F) U-2-0S cells were transfected with the indicated siRNAs and exposed to $4 \mathrm{mM} \mathrm{HU}$ for 5 hours. IdU/CldU ratios are given. (G) $\mathrm{U}-2-0 \mathrm{~S}$ cells were transfected with the indicated siRNAs and exposed to $4 \mathrm{mM} \mathrm{HU}$ for 5 hours. IdU/CldU ratios are given. (H) U-2-OS cells were transfected with the indicated siRNAs and exposed to $4 \mathrm{mM} \mathrm{HU}$ for 5 hours. IdU/CldU ratios are given. (I) Schematic model for the role of CtIP at stalled forks. CtIP regulates RAD51 stability at stalled forks, counteracting the dissolution of the RAD51 filament by FBH1. Loss of CtIP leads to DNA damage accumulation and enhanced chromosomal instability.

reads, 10 counts, and a frequency of $6.25 \%$ (corresponding to a variant detection rate of $25 \%$ per sample). Variant filtering was performed using Ingenuity Variant Analysis.

Sanger sequencing. All nonpolymorphic RBBP8 variants identified by sequencing of the 2 patient groups were verified by PCR and Sanger sequencing (for primer sequences, see Supplemental Table 4).

Statistical analysis of allelic association with breast cancer. Fisher's exact test was used to determine whether identified $R B B P 8$ variants were enriched in the examined breast cancer patient cohorts compared with the 2000 Danes used as controls in the statistical analysis.

Cell culture. The human cancer cell lines were cultured for 5 days at $37^{\circ} \mathrm{C}$ and $5 \% \mathrm{CO}_{2}$ as follows: The human breast cancer cells (MCF7) were cultured in RPMI medium (GIBCO, Life Technologies) supplemented with 10\% FBS (MilliporeSigma) and 1\% penicillin/streptomycin (GIBCO, Life Technologies). The human osteosarcoma cell line (U-2-OS) harboring inducible GFP-tagged siRNA-resistant CtIP was grown in DMEM with 10\% tetracycline-free FBS (Clontech), 1\% penicillin/streptomycin (GIBCO, Life Technologies), $100 \mu \mathrm{g} / \mathrm{mL}$ zeocin (Invitrogen), and $5 \mu \mathrm{g} / \mathrm{mL}$ blasticidin (Invitrogen). The HEK293FT cells were grown in DMEM with 10\% FBS (MilliporeSigma) and 1\% penicillin/streptomycin (GIBCO, Life Technologies).

Lentiviral infection. The doxycycline-inducible stable U-2-OS cell lines expressing the pcDNA4/TO-tagged siRNA-resistant versions of WT and mutant CtIP were established by cloning of CtIP cDNA into pcDNA4/TO-GFP vector (Invitrogen). The $\Delta \mathrm{C}$ truncation of CtIP is lacking amino acids 790-897.

The GFP-CtIP plasmids were subcloned into pLVX-TetOne Vector (Clontech) and were cotransfected with Pax8 (Clontech) and VSVG (Clontech) into HEK293FT cells using FugeneHD (Promega). The generated CtIP lentivirus was then transduced into U-2-OS cells using Polybrene according to the manufacturer's protocol, resulting in cell lines expressing GFP-tagged siRNA-resistant WT-CtIP, E804del, or $\Delta \mathrm{C}$ in a Tet-on system. To induce expression of siRNA-resistant CtIP, doxycycline $(1 \mathrm{ng} / \mathrm{mL})$ was added to the medium for approximately 24 hours.
Site-directed mutagenesis. The mutant CtIP plasmids were generated by site-directed mutagenesis of the siRNA-resistant WT-CtIP. The following primers were used: CtIP R100W forward (Fw) 5'-ACTGAAGAACATATGTGGAAAAAACAGCAAG, CtIP R100W reverse (Re) 5'-CTTGCTGTTTTTTCCACATATGTTCTTCAGT, CtIP R110Q Fw 5'-GAGTTTGAAAATATCCAGCAGCAGAATCTTAAA, CtIP R110Q Re 5'-TTTAAGATTCTGCTGCTGGATATTTTCAAACTC, CtIP R185* Fw 5'-AGAACCCCCATGTCTGATACATAGAACAAA, CtIP R185* Re 5'-TTTGTTCTATGTATCAGACATGGGGGTTCT, CtIP V198M Fw 5'-AAATTGGAGCACTCTATGTGTGCAAATGAAAT, CtIP V198M Re 5'-ATTTCATTTGCACACATAGAGTGCTCCAATTT, CtIP S231R Fw 5'- CACTTATGACCAAAGACAATCTCCAATGGCC, CtIP S231R Re 5'- GGCCATTGGAGATTGTCTTTGGTCATAAGTG, CtIP E267G Fw 5'-ACTTGGTGTTCAAGGAGAATCTGAAACTC, CtIP E267G Re 5'-GAGTTTCAGATTCTCCTTGAACACCAAGT, CtIP Q272E Fw 5'-AAGAATCTGAAACTGAAGGTCCCATGAG, CtIP Q272E Re 5'-CTCATGGGACCTTCAGTTTCAGATTCTT, CtIP G331A Fw 5'-ATCTCCTGTATTTGCAGCTACCTCTAGTA, CtIP G331A Re 5'-TACTAGAGGTAGCTGCAAATACAGGAGAT, CtIP Q352P Fw 5'-CCTTCTCTTTTACCGCCTGGGAAAAAAA, CtIP Q352P Re 5'-TTTTTTTCCCAGGCGGTAAAAGAGAAGG, CtIP I369V Fw 5'-CTTTTAGCAACACTTGTGTATCTAGATTAGAAAA, CtIP I369V Re 5'-TTTTCTAATCTAGATACACAAGTGTTGCTAAAAG, CtIP L372* Fw 5'-CACTTGTATATCTAGATGAGAAAAAACTAGATCA, CtIP L372 $^{*}$ Re 5'-TGATCTAGTTTTTTCTCATCTAGATATACAAGTG, CtIP E414D Fw 5'-AATAAAAATATAagTGATTCCCTAGgTGAaCAgA, CtIP E414D Re 5'-TCTGTTCACCTAGGGAATCACTTATATTTTTATT, CtIP H456R Fw 5'-GAGGAAGAAAGTGAACGTGAAGTAAGCTGC, CtIP H456R Re 5'-GCAGCTTACTTCACGTTCACTTTCCTC, CtIP R502L Fw 5'-TTTTCAGCTATTCAGCTTCAAGAGAAAAGCCAA, CtIP R502L Re 5'-TTGGCTTTTCTCTTGAAGCTGAATAGCTGAAAA, CtIP E552D Fw 5'-ATTCCCCAGGGGATCCCTGTTCACA, CtIP E552D Re 5'-TGTGAACAGGGATCCCCTGGGGAAT, CtIP R589H Fw 5'-TTTAAAATTCCTCTACATCCACGTGAAAGTTTG, CtIP R589H Re 5'-CAAACTTTCACGTGGATGTAGAGGAATTTTAAA, CtIP Q643P Fw 5'-AAAATAAAGTCTCTACCAAACAACCAAGATGTA, CtIP Q643P Re 5'-TACATCTTGGTTGTTTGGTAGAGACTTTATTTT, CtIP E711K Fw 5'-CAAGAGCAGAAGGGAAAAAAAAGTTCAAATG, CtIP E711K Re 5'-CATTTGAACTTTTTTTTCCCTTCTGCTCTTG, CtIP E716K Fw 5'-GGGAGAAAAAAGTTCAAATAAAGAAAGAAAAATGAATG, CtIP E716K Re 5'-CATTCATTTTTCTTTCTTTATTTGAACTTTTTTCTCCC, CtIP E804del Fw 5'-GTGGTTCGgAAAAAAgAgAgAAGA...CAG, CtIP E804del Re 5'-GTGTGCCCAAGCAGTTTTCTTCTC...CAC, CtIP R805G Fw 5'-GTTCGGAAAAAAGAGGAGGGAAGAAAACTGCTTGGGC, CtIP R805G Re 5'-GCCCAAGCAGTTTTCTTCCCTCTTTTTTCCGAA, CtIP R839Q Fw 5'-CCTGCTCAAGACACCAATTCCGCTACATTCC, CtIP R839Q Re 5'-GGAATGTAGCGGAATTGGTGTCTTGAGCAGG, CtIP P847A Fw 5'-AAGGAAGATCTTGATGCTTGTCCTCGTCCAA, CtIP P847A Re 5'-TTGGACGAGGACAAGCATCAAGATCTTCCTT, CtIP R877H Fw 5'-TTGATCCTTGTCCTCATCCAAAAAGACGT, CtIP R877H Re 5'-ACGTCTTTTTGGATGAGGACAAGGATCAA, CtIP E894D Fw 5'-TCCAAAAGGCAAGGACCAGAAGACATAGACG, CtIP E894D Re 5'-CGTCTATGTCTTCTGGTCCTTGCCTTTTGGA, CtIP $\triangle \mathrm{C}$ Fw 5'-GAAAGAGAGACTAGCTAGCAAAATTTTCCTCAT, and CtIP $\triangle$ C Re 5'-ATGAGGAAAATTTTGCTAGCTAGTCTCTCTTTC. 
PfuUltra high-fidelity polymerase (Agilent) was used according to the manufacturer's protocol.

Oligonucleotides and transfection. For siRNA transfections (48 hours), Lipofectamine RNAiMAX (Invitrogen) was used according to the manufacturer's protocol. MISSION siRNA Universal Negative Control (UNC; MilliporeSigma) was used as a negative control; the oligonucleotide sequence 5'-GCUAAAACAGGAACGAAU (obtained from Microsynth) was used for knockdown of CtIP; and a mix of 2 sequences, 5'-GGGAUGUUCUUUUGAUAAA and 5'-CCAUCCAACUUACACAUGA, was used for depletion of FBH1.

Reagents. Hydroxyurea (HU; MilliporeSigma) was used at a final concentration of $4 \mathrm{mM}$ for the indicated time. Aphidicolin (MilliporeSigma) was used at a final concentration of $0.3 \mu \mathrm{M}$ for the indicated time. Furthermore, cytochalasin B (MilliporeSigma) was used at a concentration of $1 \mu \mathrm{g} / \mathrm{mL}$.

Western blotting and antibodies. Cells were lysed on ice in EBC buffer (50 mM Tris, pH 7.4, $120 \mathrm{mM} \mathrm{NaCl}, 0.5 \%$ NP-40, and 1 mM EDTA) containing protease inhibitors ( $1 \% \mathrm{vol} / \mathrm{vol}$ aprotinin, $5 \mu \mathrm{g} / \mathrm{mL}$ leupeptin, $1 \mathrm{mM}$ PMSF), phosphatase inhibitors ( $1 \mathrm{mM} \mathrm{NaF}, 10 \mathrm{mM} \beta$-glycerophosphate), and $1 \mathrm{mM}$ DTT. The lysates were sonicated using a digital sonifier (102C CE Converter, Branson), followed by centrifugation at 20,000 $g$ for 15 minutes. Proteins were resolved by SDS-PAGE and transferred to a nitrocellulose membrane. The membrane was incubated with primary antibody followed by incubation with secondary antibody (HRP-conjugated anti-mouse or -rabbit IgG; Vector Laboratories). Immunoblots were performed using the following antibodies: CtIP (catalog A300488A, Bethyl Laboratories), RAD51 (catalog 8349, Santa Cruz Biotechnology), RAD51 (catalog 63801, Abcam), PCNA (catalog 18197, Abcam), FBH1 (FBXO18, catalog 81563, Santa Cruz Biotechnology), GFP (catalog 1181446000, Roche), actin (catalog AB1501, Merck), RPA2 S4/8 (catalog A300245A, Biosite), RPA (catalog NA29L, Merck), vinculin (catalog V9131, Merck), BRCA2 (catalog OP95, Calbiochem), H3 (catalog 1791, Abcam), and HA (catalog MMS-101P-500, Covance).

Immunofluorescence. The cells were grown on coverslips, treated as indicated, then prepared for immunofluorescence staining. Primary antibodies used were RAD51 (1:1000; 70-001, BioAcademia Japan), GFP (1:1000; catalog 1181446000, Roche), RPA (NA29L, MilliporeSigma), and RPA2 S4/8 (A300245A, Biosite). Anti-rabbit-Alexa Fluor 647 and anti-mouse-Alexa Fluor 488 (1:2000; A21245, A11017, Life Technologies) were used as secondary antibodies. For RAD51, immunofluorescence cells were pre-extracted twice for 3 minutes in CSK buffer (0.5\% Triton X-100, 20 mM HEPES, pH 7.4, 100 mM NaCl, 3 mM $\mathrm{MgCl}_{2}$, and $300 \mathrm{mM}$ sucrose) followed by fixation in $4 \%$ paraformaldehyde (VWR). Cells were permeabilized in 0.5\% Triton X-100 followed by incubation in blocking buffer (1\% BSA, $0.15 \%$ glycine, $0.1 \%$ Triton $\mathrm{X}-100)$ in PBS wash buffer (1× PBS, 0.1\% Tween-20, $1 \mathrm{mM} \mathrm{CaCl}_{2}, 0.5$ $\mathrm{mM} \mathrm{MgCl} 2$ ). Primary antibody was incubated for 1 hour at room temperature in blocking buffer, followed by 3 washes with PBS wash buffer. Secondary antibody was incubated for an additional hour, washed 3 times with PBS wash buffer, and mounted in mounting Vectashield with DAPI (Vector Laboratories). EdU staining was done per the manufacturer's instructions (Life Technologies). $Z$-stack images were acquired on a confocal Zeiss LSM 510 META microscope workstation, and images were processed and foci enumerated using Fiji (ImageJ, NIH).

Micronuclei assay. Cells were cultured on coverslips after transfection and were incubated for another 24 hours before the treatment of the cells was started. Cells were treated with aphidicolin
(Sigma-Aldrich) for 16 hours or HU (MilliporeSigma) for 5 hours. In addition, cells were treated with cytochalasin B (MilliporeSigma) for 36 hours (MCF7), to inhibit cytokinesis, and then fixed in $4 \%$ paraformaldehyde (VWR). Next, the cells were permeabilized with $0.25 \%$ Triton X-100 solution, washed twice with $1 \times$ PBS, and mounted in Vectashield with DAPI (Vector Laboratories). Binucleated cells with micronuclei were counted manually using a confocal Zeiss LSM 510 META microscope and a scanR workstation (Olympus).

Homologous recombination assay. U-2-OS cells were transfected with CtIP siRNA followed by transfection of guide RNAs targeting the lamin A (LMNA) locus and the Ruby Donor plasmid as described in Pinder et al. (33) together with empty vector or siRNA-resistant WT, E804del, or $\triangle \mathrm{C}$ CtIP. After 48 hours, LMNA genes were monitored by microscopy.

iPOND. Doxycycline-inducible U-2-OS cells were transfected with UNC (negative control) or CtIP siRNA, and 24 hours later, cells were induced with doxycycline for 24 hours. Cells were incubated with 10 $\mu \mathrm{M}$ EdU for 15 minutes, washed in media, then incubated with media containing $4 \mathrm{mM}$ HU for 5 hours, cross-linked with 1\% formaldehyde, harvested, and permeabilized. Biotin azide was covalently attached to EdU within newly replicated DNA using a click reaction, and EdU containing DNA was precipitated using streptavidin agarose beads. Eluted proteins were then analyzed by SDS-PAGE and Western blot.

DNA fibers. DNA fiber assay was carried out as described previously (22). Twenty-four hours after siRNA transfection, cells were treated with doxycycline to induce CtIP expression and left for a further 24 hours. Cells were then pulse-labeled with CldU and IdU for 20 minutes each before 5 hours of exposure to $4 \mathrm{mM} \mathrm{HU}$. At least 200 replication forks were analyzed per condition. Tract lengths were measured using Fiji, and ratios were calculated.

Proximity ligation assay on nascent DNA. Twenty-four hours after siRNA transfection, cells were treated with doxycycline to induce CtIP expression and left for a further 24 hours. Cells were then pulselabeled with $10 \mathrm{mM}$ EdU for 10 minutes followed by $4 \mathrm{mM}$ HU for 5 hours. After the indicated treatment, cells were pre-extracted for 5 minutes in buffer (0.5\% Triton X-100, 10 mM PIPES, pH 6.8, 20 mM $\mathrm{NaCl}, 3 \mathrm{mM} \mathrm{MgCl}, 300 \mathrm{mM}$ sucrose) followed by fixation in $4 \%$ paraformaldehyde (VWR). Cells were incubated in blocking buffer (3\% BSA in PBS with $0.1 \%$ sodium azide) for 1 hour at room temperature or overnight in the cold room. After blocking, cells were subjected to click reaction with biotin azide for 30 minutes and incubated overnight with the 2 relevant primary antibodies at $4^{\circ} \mathrm{C}$. The primary antibodies were diluted in PBS with 3\% FCS. The primary antibodies used were rabbit polyclonal anti-biotin (1:500; catalog A150-109A, Bethyl), mouse monoclonal anti-biotin (1:500; catalog 200-002-211, Jackson ImmunoResearch Laboratories), and rabbit polyclonal anti-CtIP (1:500; catalog A300-266A, Bethyl). The proximity ligation assay (PLA) reaction (Duolink, MilliporeSigma) to detect anti-biotin antibodies used was performed according to manufacturer instructions.

Immunoprecipitation. Extracts for immunoprecipitation were prepared using immunoprecipitation buffer (50 mM HEPES, pH 7.5, $150 \mathrm{mM} \mathrm{NaCl}, 1 \mathrm{mM}$ EDTA, $2.5 \mathrm{mM}$ EGTA, 10\% glycerol, $0.1 \%$ Tween) with protease inhibitors. Following preclearing with IgG-coupled protein $\mathrm{G}$ beads (GE Healthcare), the lysates were incubated with monoclonal anti-HA (Covance), and complexes were captured using Protein G Sepharose beads (GE Healthcare) for 2 nights at $4^{\circ} \mathrm{C}$ on a rotator. The beads were washed 5 times followed by elution of bound proteins in Laemmli sample buffer. 
PARPi sensitivity assay. Doxycycline-inducible U-2-OS cells were seeded onto CellCarrier-384 Ultra Microplates (PerkinElmer), and reverse transfection was performed using Lipofectamine RNAiMAX per the manufacturer's recommendation. After 24 hours, DMSO and different concentrations of talazoparib (BMN 673, Axon Medchem) were added to the respective wells. On day 3, DMSO and PARPicontaining media were replenished. At day 5 , CellTiter-Glo (Promega) was used to quantify the number of viable cells per the manufacturer's recommendation. Surviving fractions were calculated relative to DMSO-exposed cells for each PARPi concentration.

Statistics. Normal distribution was assessed for all experiments. Micronuclei data were normally distributed and were subsequently analyzed using 1-way ANOVA and Dunnett's multiple-comparison testing, comparing all variants with WT-CtIP-GFP. The PARPi data (Supplemental Figure $2 \mathrm{H}$ ) were Johnson-transformed, and the obtained and normally distributed data were fitted with a linear mixed model, with replicates as random effect. Multiple comparisons were performed with the lsmeans/difflsmeans and the contrast function of the lmer package in R (https://www.rdocumentation.org/packages/lme4/ versions/1.1-23/topics/lmer). Significant codes shown are comparing siCtIP and siBRCA2 with the negative control (siUNC). Foci counts, immunofluorescence intensities, and HRR data were not normally distributed. Therefore, ranks were assigned to all data from 3 biologically independent replicates, based on the number of foci/immunofluorescence intensity. The obtained ranks were used to fit a linear mixed model. $P$ values were adjusted using the Holm method if more than 2 comparisons were made. Biologically relevant $P$ values are reported with the following significance codes: ${ }^{* *} P<0.0001,{ }^{* *} P<0.001,{ }^{*} P<$ 0.05. All graphs represent the mean (red line) \pm SEM (black).

Study approval. The study was approved by the Capital Region of Denmark (H-4-2010-050), the Danish Data Protection Agency (RH2016-353, I-Suite no. 05097), and the DBCG (jr. no. DBCG-2013-15).

\section{Author contributions}

RZ designed and performed the cell biology experiments and iPOND experiment. MRH designed and performed the DNA fiber assay. KV performed micronuclei and HRR assays. BE diagnosed and enrolled the breast cancer patients. MR, BB, and FCN performed sequencing and data analysis. ANK generated an inducible complementation system in U-2-OS cells. HR designed the cell biology experiments. MB performed the PARPi experiments. RZ, BB, MRH, MR, FCN, GSS, and CSS wrote the manuscript. The study was planned and supervised by GSS, FCN, and CSS. Order of the co-first authors was based on (a) experimental data and conceptual contributions, (b) duration in time of work on the project, and (c) contributions during revision processes. RZ contributed for a longer time period and was more involved at early conceptual stages compared with KV and MRH. MRH contributed for an overall extended time period and was more involved at early conceptual stages compared with $\mathrm{KV}$.

\section{Acknowledgments}

We thank the COMPLEXO Network for data access and advice (see Supplemental Acknowledgments for consortium details). CSS is funded by the Danish Cancer Society, Danish Medical Research Council, and Lundbeck Foundation. CSS and KVO have received funding from the European Union's Horizon 2020 research and innovation program under the Marie Sklodowska-Curie grant (agreement number 722729). MRH is funded by an MRC Career Development Fellowship (MR/P009085/1) and a Birmingham Fellowship awarded by the University of Birmingham. GSS is funded by a CR-UK Programme Grant (C17183/A23303). FCN is funded by the Lundbeck Foundation and Research Council of the Capital Region of Denmark. COMPLEXO is supported by a program grant from the National Health and Medical Research Council of Australia (APP1074383). We thank Alex Sartori (University of Zurich, Zurich, Germany) for sharing unpublished data with us.

Address correspondence to: Finn Cilius Nielsen. Phone: 0045.3545.2223; Email: finn.cilius.nielsen@regionh.dk. Or to: Claus S. Sørensen. Phone: 0045.3532.5678; Email: claus.storgaard@bric.ku.dk.
1. Nielsen FC, van Overeem Hansen T, Sørensen CS. Hereditary breast and ovarian cancer: new genes in confined pathways. Nat Rev Cancer. 2016;16(9):599-612.

2. Sun J, et al. Mutations in RECQL gene are associated with predisposition to breast cancer. PLOS Genet. 2015;11(5):e1005228.

3. Cybulski C, et al. Germline RECQL mutations are associated with breast cancer susceptibility. Nat Genet. 2015;47(6):643-646.

4. Santos-Pereira JM, Aguilera A. R loops: new modulators of genome dynamics and function. Nat Rev Genet. 2015;16(10):583-597.

5. Schlacher K, Christ N, Siaud N, Egashira A, Wu H, Jasin M. Double-strand break repair-independent role for BRCA2 in blocking stalled replication fork degradation by MRE11. Cell. 2011;145(4):529-542.

6. Ray Chaudhuri A, et al. Replication fork stability confers chemoresistance in BRCA-deficient cells. Nature. 2016;535(7612):382-387.

7. Couch FJ, Nathanson KL, Offit K. Two decades after BRCA: setting paradigms in personalized cancer care and prevention. Science. 2014;343(6178):1466-1470.

8. Foo TK, et al. Compromised BRCA1-PALB2 interaction is associated with breast cancer risk. Oncogene. 2017;36(29):4161-4170.

9. Seal S, et al. Truncating mutations in the Fanconi anemia J gene BRIP1 are low-penetrance breast cancer susceptibility alleles. Nat Genet. 2006;38(11):1239-1241.

10. Rahman N, et al. PALB2, which encodes a BRCA2-interacting protein, is a breast cancer susceptibility gene. Nat Genet. 2007;39(2):165-167.

11. Golmard L, et al. Germline mutation in the RAD51B gene confers predisposition to breast cancer. BMC Cancer. 2013;13:484.

12. Lohmueller KE, et al. Whole-exome sequencing of 2,000 Danish individuals and the role of rare coding variants in type 2 diabetes. Am J Hum Genet. 2013;93(6):1072-1086.

13. Bunting SF, et al. 53BP1 inhibits homologous recombination in Brca1-deficient cells by blocking resection of DNA breaks. Cell. 2010;141(2):243-254.
14. Gossen M, Bujard H. Tight control of gene expression in mammalian cells by tetracyclineresponsive promoters. Proc Natl Acad Sci US A. 1992;89(12):5547-5551.

15. Sartori AA, et al. Human CtIP promotes DNA end resection. Nature. 2007;450(7169):509-514.

16. Przetocka S, et al. CtIP-mediated fork protection synergizes with BRCA1 to suppress genomic instability upon DNA replication stress. Mol Cell. 2018;72(3):568-582.e6.

17. Raderschall E, Golub EI, Haaf T. Nuclear foci of mammalian recombination proteins are located at single-stranded DNA regions formed after DNA damage. Proc Natl Acad Sci U S A. 1999;96(5):1921-1926.

18. Schlacher K, Wu H, Jasin M. A distinct replication fork protection pathway connects Fanconi anemia tumor suppressors to RAD51-BRCA1/2. Cancer Cell. 2012;22(1):106-116.

19. Sirbu BM, Couch FB, Cortez D. Monitoring the spatiotemporal dynamics of proteins at replication forks and in assembled chromatin using isolation of proteins on nascent DNA. Nat Protoc. 
2012;7(3):594-605.

20. Petruk $S$, et al. TrxG and PcG proteins but not methylated histones remain associated with DNA through replication. Cell. 2012;150(5):922-933.

21. Taglialatela A, et al. Restoration of replication fork stability in BRCA1- and BRCA2-deficient cells by inactivation of SNF2-family fork remodelers. Mol Cell. 2017;68(2):414-430.e8.

22. Higgs MR, et al. BOD1L is required to suppress deleterious resection of stressed replication forks. Mol Cell. 2015;59(3):462-477.

23. Soria-Bretones I, Sáez C, Ruíz-Borrego M, Japón MA, Huertas P. Prognostic value of CtIP/ RBBP8 expression in breast cancer. Cancer Med. 2013;2(6):774-783.

24. Simandlova J, et al. FBH1 helicase disrupts RAD51 filaments in vitro and modulates homologous recombination in mammalian cells. J Biol Chem. 2013;288(47):34168-34180.
25. Higgs MR, et al. Histone methylation by SETD1A protects nascent DNA through the nucleosome chaperone activity of FANCD2. Mol Cell. 2018;71(1):25-41.e6.

26. Wang J, Ding Q, Fujimori H, Motegi A, Miki Y, Masutani M. Loss of CtIP disturbs homologous recombination repair and sensitizes breast cancer cells to PARP inhibitors. Oncotarget. 2016;7(7):7701-7714.

27. Lin ZP, Ratner ES, Whicker ME, Lee Y, Sartorelli AC. Triapine disrupts CtIP-mediated homologous recombination repair and sensitizes ovarian cancer cells to PARP and topoisomerase inhibitors. Mol Cancer Res. 2014;12(3):381-393.

28. Gorringe KL, Choong DY, Lindeman GJ, Visvader JE, Campbell IG. Breast cancer risk and the BRCA1 interacting protein CTIP. Breast Cancer Res Treat. 2008;112(2):351-352.

29. Bonache S, et al. Multigene panel testing beyond
BRCA1/2 in breast/ovarian cancer Spanish families and clinical actionability of findings. J Cancer Res Clin Oncol. 2018;144(12):2495-2513.

30. Chen PL, et al. Inactivation of CtIP leads to early embryonic lethality mediated by G1 restraint and to tumorigenesis by haploid insufficiency. $\mathrm{Mol}$ Cell Biol. 2005;25(9):3535-3542.

31. Reczek CR, Shakya R, Miteva Y, Szabolcs M, Ludwig T, Baer R. The DNA resection protein CtIP promotes mammary tumorigenesis. Oncotarget. 2016;7(22):32172-32183.

32. Li H, Durbin R. Fast and accurate long-read alignment with Burrows-Wheeler transform. Bioinformatics. 2010;26(5):589-595.

33. Pinder J, Salsman J, Dellaire G. Nuclear domain 'knock-in' screen for the evaluation and identification of small molecule enhancers of CRISPR-based genome editing. Nucleic Acids Res. 2015;43(19):9379-9392. 